\title{
THE GENERALIZED LUSTERNIK-SCHNIRELMANN CATEGORY OF A PRODUCT SPACE
}

\author{
MÓNICA CLAPP AND DIETER PUPPE
}

\begin{abstract}
We continue to study the notions of $\mathscr{A}$-category and strong $\mathscr{A}$ category which we introduced in [2]. We give a characterization of them in terms of homotopy colimits and then use it to prove some product theorems in this context.
\end{abstract}

In [2] we introduced new homotopy invariants by generalizing the notions of Lusternik-Schnirelmann category and strong category as follows:

For any given class of spaces $\mathscr{A}$ we define the $\mathscr{A}$-category $\mathscr{A}$-cat $(X)$ of a space $X$ to be the smallest integer $k$ for which there exists a numerable covering $\left(X_{1}, \ldots, X_{k}\right)$ of $X$ such that each inclusion $X_{j} \subset X$ factors through some space $A_{j} \in \mathscr{A}$ up to homotopy. If no such covering exists we set $\mathscr{A}$-cat $(X):=$ $\infty . \mathscr{A}-\operatorname{cat}(X)$ is an invariant of the homotopy type of $X[2,1.4]$.

If we replace the condition that each $X_{j} \subset X$ factors through some space in $\mathscr{A}$ up to homotopy by asking each $X_{j}$ to have the homotopy type of some space in $\mathscr{A}$, then the number $\mathscr{A}-\operatorname{gcat}(X)$ thus obtained is not a homotopy invariant [3]. So we define the strong $\mathscr{A}$-category $\mathscr{A}$-Cat $(X)$ of $X$ to be the minimum of $\mathscr{A}-\operatorname{gcat}\left(X^{\prime}\right)$ for all spaces $X^{\prime}$ having the homotopy type of $X$.

If $\mathscr{A}$ consists only of the one-point-space then $\mathscr{A}$-cat $(X)$ is just LusternikSchnirelmann's category cat $(X)$ of $X$ and $\mathscr{A}$-Cat $(X)$ is the strong category Cat $(X)$ introduced by Ganea in [5]. Other interesting examples are the $q$ connective (strong) category cat $_{q}(X) \quad\left(\mathrm{Cat}_{q}(X)\right)$ of $X$ obtained by taking $\mathscr{A}$ to be the class of $q$-connected $\mathrm{CW}$-complexes, $q \geq 0$, and the $q$-dimensional (strong) category cat $^{q}(X)\left(\mathrm{Cat}^{q}(X)\right)$ of $X$ obtained by taking for $\mathscr{A}$ the class of all $\mathrm{CW}$-complexes of dimension $\leq q[2,1.2]$.

In this paper we shall prove the following product theorems, which generalize the well known ones for the classical case [7]. Unlike [7] however, we do not restrict ourselves to polyhedra but rather exploit the linear structure given by the numeration of the coverings.

Received by the editors November 11, 1986.

1980 Mathematics Subject Classification (1985 Revision). Primary 55M30; Secondary 55P50.

Key words and phrases. Lusternik-Schnirelmann category, strong category, homotopy colimits.

The first author wishes to thank the University of Heidelberg for its hospitality and financial support. 
Theorem 1. Let $\mathscr{A}, \mathscr{B}$ and $\mathscr{C}$ be classes of spaces such that

(a) $A \times B \in \mathscr{C}$ for all $A \in \mathscr{A}, B \in \mathscr{B}$,

(b) $\mathscr{C}$ contains all finite wedges of elements in $\mathscr{C}$.

Then, for any two path-connected spaces $X$ and $Y$,

$$
\mathscr{C}-\operatorname{cat}(X \times Y) \leq \mathscr{A}-\operatorname{cat}(X)+\mathscr{B}-\operatorname{cat}(Y)-1
$$

and

$$
\mathscr{C}-\operatorname{Cat}(x \times Y) \leq \mathscr{A}-\operatorname{Cat}(X)+\mathscr{B}-\operatorname{Cat}(Y)-1 .
$$

Theorem 2. Let $X$ and $Y$ be pathwise connected spaces and let $\mathscr{A}$ be a class of spaces which contains all finite wedges of elements in it. Then

$$
\mathscr{A}-\operatorname{Cat}(X \times Y) \leq \mathscr{A}-\operatorname{cat}(X)+\max \{\operatorname{Cat}(Y), 2\}-1 .
$$

\section{CATEGORY AND hOMOTOPY COLIMITS}

Let $K$ be a simplicial complex and let $K$ also denote the poset of its simplices ordered by opposite inclusion. For any functor $F: K \rightarrow$ Top, the homotopy colimit $h$-colim $F$ of $F$ may be defined as the quotient space of the disjoint union

$$
\bigsqcup_{\sigma \in K}\left(F(\sigma) \times \Delta_{\sigma}\right)
$$

obtained by identifying $\left(x, \varepsilon_{\tau}^{\sigma} t\right)$ with $\left(f_{\sigma}^{\tau} x, t\right)$ for all $x \in F(\tau), t \in \Delta_{\sigma}$, where $\Delta_{\sigma}$ is the geometric simplex spanned by $\sigma, \varepsilon_{\tau}^{\sigma}: \Delta_{\sigma} \rightarrow \Delta_{\tau}$ is the face map induced by $\sigma \subset \tau$, and $f_{\sigma}^{\tau}: F(\tau) \rightarrow F(\sigma)$ is the image of $\tau \leq \sigma$ under the functor $F$. The images $F(v)$ of the vertices of $K$ are called the vertex-spaces of $F$ or of $h$-colim $F$.

A $k$-fold mapping cylinder is the homotopy colimit of a functor $F: \Delta^{k-1} \rightarrow$ Top, where $\Delta^{k-1}$ is the standard $(k-1)$-simplex $[2,5.1]$.

Given a covering $U=\left(X_{0}, \ldots, X_{k-1}\right)$ of a space $X$ we associate to it the functor $U: \Delta^{k-1} \rightarrow X$ such that

$$
U(\sigma)=\bigcap_{i \in \sigma} X_{i} \text { and } U(\tau \leq \sigma): U(\tau) \subset U(\sigma) .
$$

The homotopy colimit of $U$ is known as the classifying space $B U$ of $U$ and the canonical map $B U \rightarrow X$ is a homotopy equivalence if $U$ is, for example, numerable [4].

On the other hand, for any functor $F: K \rightarrow$ Top, there is a canonical map

$$
p: h \text {-colim } F \rightarrow K \text {. }
$$

The inverse images under $p$ of the open stars $\operatorname{St}(v)$ of the vertices $v$ of $K$ form a numerable covering of $h$-colim $F$ and the canonical contraction of $\operatorname{St}(v)$ to $v$ lifts canonically to a fiberwise strong deformation retraction of $p^{-1} \mathrm{St}(v)$ to $p^{-1}(v)=F(v)$. 
The discussion above shows in particular that

Proposition 3 [2, 5.2]. For every class of spaces $\mathscr{A}, \mathscr{A}$-Cat $(X) \leq k$ if and only if $X$ has the homotopy type of a $k$-fold mapping cylinder with vertex-spaces in $\mathscr{A}$.

For every class of spaces $\mathscr{C}$, let $h \mathscr{C}$ denote the class of spaces having the homotopy type of some space in $\mathscr{C}$. The "if" part of the proposition above can be improved as follows:

Proposition 4. Let $\mathscr{C}$ be a class of spaces and let $F: K \rightarrow$ Top be a functor with vertex-spaces in $h \mathscr{C}$. Assume that one of the following conditions holds:

(a) $h \mathscr{C}$ contains all finite disjoint unions of elements in $h \mathscr{C}$, or

(b) $h$-colim $F$ is pathwise connected and $h \mathscr{C}$ contains all finite wedges of elements in $h \mathscr{C}$. Then

$$
\mathscr{C}-\operatorname{Cat}(h-\operatorname{colim} F) \leq 1+\operatorname{dim} K .
$$

Proof. Let $k=1+\operatorname{dim} K$. Since at most $k$ open stars have a nonempty intersection one can construct a partition $V_{1}, \ldots, V_{k}$ of the vertices of $K$ such that, for each $j=1, \ldots, k$, the family $\left(\operatorname{St}(v) \mid v \in V_{j}\right)$ is disjoint. Hence

$$
U_{j}:=p^{-1}\left(\bigcup_{v \in V_{j}} \operatorname{St}(v)\right)=\bigsqcup_{v \in V_{j}} p^{-1} \operatorname{St}(v) .
$$

Since $F(v)$ is a strong deformation retract of $p^{-1} \operatorname{St}(v), p^{-1} \operatorname{St}(v) \in h \mathscr{C}$. If condition (a) holds we are done. If condition (b) holds we now apply the following lemma.

Lemma 5. Let $X$ be a path-connected space and let $\left(Y_{1}, Y_{2}, X_{1}, \ldots, X_{k}\right)$ be a numerable covering of $X$ such that $Y_{1} \cap Y_{2}=\varnothing$. Then there is a space $X^{\prime}$ of the homotopy type of $X$ and a numerable covering $\left(W, X_{1}^{\prime}, \ldots, X_{k}^{\prime}\right)$ of $X^{\prime}$ such that $W \simeq Y_{1} \vee Y_{2}$ and $X_{i}^{\prime} \simeq X_{i}$ for all $i=1, \ldots, k$.

Proof. Replacing $X$ by the classifying space of the given covering if necessary we may assume that the covering is closed. Hence, since $X$ is path-connected, there is a path $\omega:[0,1] \rightarrow X$ such that $\omega(0) \in Y_{1}, \omega(1) \in Y_{2}$ and $\omega(0,1) \in$ $X-\left(Y_{1} \cup Y_{2}\right)$. Now just take $X^{\prime}:=X \times[0,1], X_{i}^{\prime}=X_{i} \times[0,1], i=1, \ldots, k$, and

$$
W:=\left(Y_{1} \times I\right) \cup\{(\omega(t), t) \mid 0 \leq t \leq 1\} \cup\left(Y_{2} \times I\right) .
$$

Corollary 6. Let $\mathscr{C}$ be a class of spaces and let $X$ be a space such that $\mathscr{C}-\operatorname{Cat}(X)$ $=k$. Assume that either

(a) $h \mathscr{C}$ contains all finite disjoint unions of elements in $h \mathscr{C}$, or

(b) $X$ is pathwise connected and $h \mathscr{C}$ contains all finite wedges of elements in $h \mathscr{C}$. 
Then every numerable covering $U=\left(X_{1}, \ldots, X_{k}\right)$ of $X$ with $X_{i} \in h \mathscr{C}$ has a nonempty intersection.

Proof. Assume $X_{1} \cap \cdots \cap X_{k}=\varnothing$. Then the classifying space $B U$ of $U$ is the homotopy colimit of the restriction of the functor $U$ to the boundary of $\Delta^{k-1}$. Hence, by Proposition 4, $\mathscr{C}-\operatorname{Cat}(X)=\mathscr{C}-\operatorname{Cat}(B U) \leq k-1$.

We are now ready to prove Theorem 1. It is a particular case of the following more general Theorems 7 and $7^{\prime}$.

Theorem 7. Let $\mathscr{A}, \mathscr{B}$ and $\mathscr{C}$ be classes of spaces and $X$ and $Y$ be spaces such that $A \times B \in h \mathscr{C}$ for all $A \in \mathscr{A}, B \in \mathscr{B}$ and such that one of the following conditions holds:

(a) $h \mathscr{C}$ contains all finite disjoint unions of elements of $h \mathscr{C}$, or

(b) $X$ and $Y$ are path-connected and hE् contains all finite wedges of elements in $h \mathscr{C}$.

Then

$$
\mathscr{C}-\operatorname{Cat}(X \times Y) \leq \mathscr{A}-\operatorname{Cat}(X)+\mathscr{B}-\operatorname{Cat}(Y)-1 .
$$

Proof. Let $k=\mathscr{A}$-Cat $(X)$ and $n=\mathscr{B}-\operatorname{Cat}(Y)$. By Proposition 3, we may assume that $X$ and $Y$ are the homotopy colimits of functors $U: \Delta^{k-1} \rightarrow$ Top and $V: \Delta^{n-1} \rightarrow$ Top with vertex-spaces in $\mathscr{A}$ and $\mathscr{B}$ respectively. Consider the canonical simplicial subdivision $K$ of $\Delta^{k-1} \times \Delta^{n-1}$ and define a functor $F: K \rightarrow$ Top by

$$
F(\rho)=U(\sigma) \times V(\tau)
$$

where $\sigma$ and $\tau$ are the smallest simplices of $\Delta^{k-1}$ and $\Delta^{n-1}$ respectively such that $\rho \subset \sigma \times \tau$. Then $X \times Y$ is homeomorphic to $h$-colim $F$ and the result follows from Proposition 4.

It is easy to show $[2,5.5]$ that, for every class of spaces $\mathscr{A}, \mathscr{A}$-cat $(X) \leq k$ if and only if $X$ is dominated by a space $Z$ such that $\mathscr{A}$-Cat $(Z) \leq k$. Hence, if the inequality in Theorem 7 holds, then

$$
\mathscr{C}-\operatorname{cat}(X \times Y) \leq \mathscr{A}-\operatorname{cat}(X)+\mathscr{B}-\operatorname{cat}(Y)-1
$$

also holds, and this proves Theorem 1 . This last inequality holds however under even weaker assumptions. There are in fact analogous results to the ones given above (with weaker assumptions) for $\mathscr{A}$-cat. We state them below without proofs. These are completely analogous to the given ones for $\mathscr{A}$-Cat.

Proposition $3^{\prime}$. For every class of spaces $\mathscr{A}, \mathscr{A}-\operatorname{cat}(X) \leq k$ if and only if $X$ has the homotopy type of a $k$-fold mapping cylinder $Z$ such that the inclusion $Z_{v} \subset$ $Z$ of each vertex-space $Z_{v}$ factors through some space in $\mathscr{A}$ up to homotopy. 
We shall say that a class $\mathscr{C}$ satisfies condition $(*)$ for a space $X$ if the following holds

(*) For each finite family of maps $f_{i}: C_{i} \rightarrow X$ with $C_{i} \in \mathscr{C}$, the induced map on the disjoint union of the $C_{i}$ 's,

$$
\left\{f_{i}\right\}: \bigsqcup_{i} C_{i} \rightarrow X
$$

factors through some space in $\mathscr{C}$ up to homotopy.

This condition is satisfied if, for example, there is a $\mathscr{C}$-universal map $u: U \rightarrow$ $X$ in the sense of $[2,4.1]$. Observe that conditions (a) and (b) above imply condition $(*)$ for the corresponding spaces.

Proposition $4^{\prime}$. Let $\mathscr{C}$ be a class of spaces and let $F: K \rightarrow$ Top be a functor such that the inclusion $F(v) \subset h$-colim $F$ of each vertex-space $F(v)$ factors through some space in $\mathscr{C}$ up to homotopy. Assume further that $\mathscr{C}$ satisfies condition (*) for $h$-colim $F$. Then

$$
\mathscr{C}-\operatorname{cat}(h-\operatorname{colim} F) \leq 1+\operatorname{dim} K .
$$

Corollary $6^{\prime}$. If $\mathscr{C}-\operatorname{cat}(C)=k$ and $\mathscr{C}$ satisfies condition $(*)$ for $X$, then every numerable covering $\left(X_{1}, \ldots, X_{k}\right)$ of $X$ such that the inclusion $X_{i} \subset X$ factors through some space in $\mathscr{C}$ up to homotopy for all $i=1, \ldots, k$, has a nonempty intersection.

Theorem $7^{\prime}$. Let $\mathscr{A}, \mathscr{B}$ and $\mathscr{C}$ be classes of spaces such that $A \times B$ is dominated by some $C \in \mathscr{C}$ for all $A \in \mathscr{A}, B \in \mathscr{B}$ and let $X$ and $Y$ be spaces such that $\mathscr{C}$ satisfies condition $(*)$ for $X \times Y$. Then

$$
\mathscr{C}-\operatorname{cat}(X \times Y) \leq \mathscr{A}-\operatorname{cat}(X)+\mathscr{B}-\operatorname{cat}(Y)-1 .
$$

Applying these results to the particular examples mentioned in the introduction we obtain

Corollary 8. If $X$ and $Y$ are path-connected then

$$
\operatorname{Cat}(X \times Y) \leq \operatorname{Cat}(X)+\operatorname{Cat}(Y)-1 \quad(c f .[7])
$$

and

$$
\mathrm{Cat}_{\min (p, q)}(X \times Y) \leq \mathrm{Cat}_{q}(X)+\mathrm{Cat}_{p}(Y)-1 .
$$

The analogous inequalities for cat and cat ${ }_{k}$ also hold.

Corollary 9. For all $p, q \geq 0$ and all spaces $X$ and $Y$,

$$
\mathrm{Cat}^{p+q}(X \times Y) \leq \mathrm{Cat}^{p}(X)+\mathrm{Cat}^{q}(Y)-1 .
$$

and analogously for $\mathrm{cat}^{k}$. 


\section{THE MIXED PRODUCT THEOREM}

Following Takens' ideas [7] we shall now prove

Theorem 10. Let $X$ and $Y$ be spaces such that $Y$ is path-connected and $\operatorname{Cat}(Y)$ $>1$ and let $\mathscr{C}$ be a class of spaces which satisfies condition $(*)$ for $X$. Then

$$
\mathscr{C}-\operatorname{Cat}(X \times Y) \leq \mathscr{C} \text { - } \operatorname{cat}(X)+\operatorname{Cat}(Y)-1
$$

Proof. Let $k=\mathscr{C}-\operatorname{cat}(X)$ and $n=\operatorname{Cat}(Y), n \geq 2$. We may assume that $Y$ is an $n$-fold mapping cylinder whose vertex-spaces are points and we denote by $p: Y \rightarrow \Delta^{n-1}$ the canonical map. Let $S_{i}(j)$ be the closed $(1-1 /(n+i+1))$-star of the $j$ th vertex of $\Delta^{n-1}, 0 \leq i \leq k-1,0 \leq j \leq n-1$, and let

$$
K_{i j}:=S_{i}(j)-\operatorname{int}\left(S_{i}(j+1) \cup \cdots \cup S_{i}(n-1)\right) .
$$

Then we have:

(1) The canonical contraction of $S_{i}(j)$ induces a contraction of $K_{i j}$.

(2) $\left(K_{i j} \mid 0 \leq j \leq n-1\right)$ is a closed covering of $\Delta^{n-1}$ for each $i$.

(3) $\left(K_{i j} \mid i+j=q\right)$ is a disjoint family for each $q=0, \ldots, k+n-2$.

Now we make $K_{i j}$ a little larger such that (keeping the same notation) the above properties (1)-(3) are still satisfied and in addition

(4) the covering $\left(K_{i j} \mid 0 \leq j \leq n-1\right)$ of $\Delta^{n-1}$ is numerable for each $i$.

Let $Y_{i j}=p^{-1} K_{i j}$. Since $n \geq 2$, there are embeddings $\omega_{i j}:[0,1] \rightarrow Y$ such that the image of $\omega_{i j}$ meets $Y_{i j}$ precisely in the point $\omega_{i j}(0)$ and, for each $0 \leq q \leq k+n-2$, the family $\left(Y_{i j} \cup \omega_{i j}[0,1] \mid i+j=q\right)$ is disjoint except for one common point which is $\omega_{i j}(1)$ for all $i+j=q$. One way of getting such embedded paths is by constructing embeddings $\omega_{i j}^{\prime}:[0,1] \rightarrow \Delta^{n-1}$ which have the analogous properties for $K_{i j}$ instead of $Y_{i j}$ and taking their images under a section of $p$, which exists because of Corollary 6 .

Let now $\left(X_{0}, \ldots, X_{k-1}\right)$ be a numerable covering of $X$ such that each inclusion $X_{i} \subset X$ factors through some space in $\mathscr{C}$ up to homotopy. Since $\mathscr{C}$ satisfies condition $(*)$ for $X$ there is a space $C \in h \mathscr{C}$ and maps $f_{i}: X_{i} \rightarrow C$ and $\alpha: C \rightarrow X$ such that $\alpha f_{i}$ is homotopic to $X_{i} \subset X$. Replacing $\alpha$ by its associated fibration we may assume that $\alpha f_{i}$ is the inclusion $X_{i} \subset X$.

Let $Z_{i}$ be the mapping cylinder of $f_{i}: X_{i} \rightarrow C$ and $Z$ be the mapping cylinder of $\left\{f_{i}\right\}: X_{0} \sqcup \cdots \sqcup X_{k-1} \rightarrow C$ (in both cases we identify $(x, 1) \in X_{i} \times I$ with $\left.f_{i}(x) \in C\right)$. The idea is now to glue $Z_{i}$ to $X_{i} \times Y_{i j}$ along $X_{i} \times 0 \subset Z_{i}$, obtaining a space which is homotopy equivalent to $C$, hence an element of $h \mathscr{C}$, and then embed it into a space of the homotopy type of $X \times Y$. More precisely we proceed as follows: Let $C Z=Z \times I / Z \times\{1\}$ be the cone of $Z$ and let $W_{i j}$ be the quotient space of the disjoint union of $X_{i} \times Y_{i j} \times C Z$ and $Z_{i}$ obtained by identifying $(x, 0) \in Z_{i}$ with $\left(x, \omega_{i j}(0), x, 0,0\right) \in X_{i} \times Y_{i j} \times C Z$ for each $x \in X_{i}$. Then, since $Y_{i j}$ and $C Z$ are contractible, $C$ is a strong deformation retract of $W_{i j}$, and there is a closed embedding

$$
W_{i j} \subset X \times Y \times C Z
$$


which is just the inclusion on $X_{i} \times Y_{i j} \times C Z$, and on $Z_{i}$ is given by

$$
(x, t) \mapsto\left(x, \omega_{i j}(t), x, t, 0\right) \text { and } c \mapsto\left(\alpha(c), \omega_{i j}(1), c, 0\right)
$$

for $x \in X_{i}, t \in[0,1], c \in C$. Let now

$$
W_{q}:=\bigcup_{i+j=q} W_{i j} .
$$

$\left(W_{q} \mid q=0, \ldots, k+n-2\right)$ is a numerable covering of $X \times Y \times C Z$ and, since $W_{i j} \cap W_{r s}=C$ if $i+j=r+s$ and $i \neq r, C$ is a strong deformation retract of $W_{q}$. But $X \times Y \times C Z \simeq X \times Y$, hence $\mathscr{C}$-Cat $(X \times Y) \leq k+n-1$.

Replacing $Y$ by $[0,1]$ and $p$ by the identity $(n=2)$ in the proof of Theorem 10 we obtain a proof of

Theorem $11[2,5.6]$. If $\mathscr{C}$ satisfies condition $(*)$ for $X$, then

$$
\mathscr{C}-\operatorname{Cat}(X) \leq \mathscr{C}-\operatorname{cat}(X)+1 \text {. }
$$

Theorem 2 is just a special case of Theorems 10 and 11 together. Both of these theorems apply to the examples we gave in the introduction. In the classical case they are just Takens' mixed product theorem [7].

For the $q$-connective categories there is actually a better result, namely

\section{Proposition 12.}

$$
\mathrm{Cat}_{\min (p, q)}(X \times Y) \leq \operatorname{cat}_{p} X+\max \left\{\mathrm{Cat}_{q} Y, 2\right\}-1 \text {. }
$$

Proof. Let $r=\min (p, q)$. Observe that the spaces $W_{i j}$ constructed in the proof of Theorem 10 are $r$-connected if $Y_{i j}$ and $C$ are $r$-connected. So the proof of Theorem 10 carries over to this situation.

Remarks. We do not know whether a better mixed product formula holds in general, that is, a formula that looks like the one in Theorem 7 with $\mathscr{A}$-cat $(X)$ instead of $\mathscr{A}$-Cat $(X)$, assuming $\mathscr{B}-\operatorname{Cat}(Y)>1$. It is also not known whether Takens' formula is the best possible, that is, whether for example

$$
\operatorname{Cat}(X \times Y) \leq \operatorname{cat}(X)+\operatorname{cat}(Y)-1
$$

also holds for $\operatorname{cat}(Y)>1$. The trouble in finding counterexamples in both cases is that one has to prove that Cat is greater than 3. In fact this is the main difficulty in finding examples of spaces $X$ such that $\operatorname{Cat}(X) \neq \operatorname{cat}(X)$, for $\operatorname{cat}(X) \geq 3[2,5.10]$. For $\operatorname{cat}(X)=2$ one has the Berstein-Hilton example $C_{\alpha}$ which is obtained by attaching a 7-cell to $S^{3}$ via the element $\alpha$ of order 3 in $\pi_{6} S^{3}$. Then $\operatorname{cat}\left(C_{\alpha}\right)=2$ but $\operatorname{Cat}\left(C_{\alpha}\right)=3[1 ; 6,2.4]$. Using the formulas above one can show that

$$
\operatorname{cat}\left(C_{\alpha} \times C_{\alpha}\right)=3 \text { and } \operatorname{Cat}\left(C_{\alpha} \times C_{\alpha}\right) \leq 4
$$

and one would of course like to have $\operatorname{Cat}\left(C_{\alpha} \times C_{\alpha}\right)=4$. Now, Hans-Werner Henn has shown (personal communication) that after localizing away from 2, $\operatorname{Cat}\left(C_{\alpha} \times C_{\alpha}\right)=3$. The unlocalized question remains open. 


\section{REFERENCES}

1. I. Berstein and P. J. Hilton, Category and generalized Hopf invariants, Illinois J. Math. 4 (1960), 437-451.

2. M. Clapp and D. Puppe, Invariants of the Lusternik-Schnirelmann type and the topology of critical sets, Trans. Amer. Math. Soc. (to appear).

3. M. Clapp and L. Montejano, Lusternik-Schnirelmann category and minimal coverings with contractible sets, Manuscripta Math. (to appear).

4. T. tom Dieck, Partitions of unity in homotopy theory, Compositio Math. 23 (1971), 159167.

5. T. Ganea, Lusternik-Schnirelmann category and strong category, Illinois J. Math. 11 (1967), 417-427.

6. L. Montejano, Lusternik-Schnirelmann category: A geometric approach, Memoirs of the Semester for Geometric and Algebraic Topology, Banach Center Publ., vol. 18, Warsaw, 1986, pp. 119-131.

7. F. Takens, The Lusternik-Schnirelmann categories of a product space, Compositio Math. 22 (1970), 175-180.

Instituto de Matemáticas, UNAM, Circuito Exterior, Ciudad Universitaria, 04510 México, D. F. Mexico

Mathematisches Institut der Universität, Im Neuenheimer Feld 288, D-6900 HeidelBerg, Germany (FEDERAL Republic) 